\title{
AFRONTAMIENTO AL ESTRÉS Y ORIENTACIÓN SUICIDA EN ESTUDIANTES ADOLESCENTES
}

\section{Coping with stress and suicide orientation in adolescent students}

\author{
Carlos Richard Chacaliaza Uribe \\ Inkamay Salud
}

\begin{abstract}
Resumen
El objetivo de la investigación fue determinar la relación existente entre el afrontamiento al estrés y la orientación suicida en estudiantes de la Institución Educativa Nuestra Señora de Las Mercedes de Ica. La muestra fue de tipo censal, conformada por 180 estudiantes femeninas, cuyas edades fluctúan entre los 15 y 17 años, del $4^{\circ}$ y $5^{\circ}$ año de nivel secundario. Los instrumentos utilizados fueron: Cuestionario de Modos de Afrontamiento al Estrés y el Inventario de Orientaciones Suicidas. Se obtuvieron los siguientes resultados: Existe una relación muy significativa y negativa entre el afrontamiento al estrés y la orientación suicida (r:-0.292). Por lo que se concluye, que a una mayor capacidad de afrontamiento al estrés los niveles de orientación suicida disminuyen.
\end{abstract}

Palabras clave: estrés, afrontamiento, orientación suicida, estudiantes

\begin{abstract}
The objective of the research was to determine the relationship between coping with stress and suicidal orientation in students of the Our Lady of Las Mercedes de Ica Educational Institution. The sample was of the census type, made up of 180 female students, whose ages range between 15 and 17 years, from the 4th and 5th year of secondary level. The instruments used were: Stress Coping Modes Questionnaire and Suicide Guidance Inventory. The following results were obtained: There is a very significant and negative relationship between coping with stress and suicidal orientation ( $r$ :-0.292). Therefore, it is concluded that at a greater capacity to cope with stress, the levels of suicidal orientation decrease.
\end{abstract}

Keywords: stress, coping, suicidal orientation, students

\footnotetext{
* Artículo basado en tesis de Licenciatura sustentada por el autor.

** Psicólogo.chaurica@gmail.com ORCID: https://orcid.org/0000-0001-6591-5226
} 


\section{INTRODUCCIÓN}

Las estudiantes se encuentran en constantes cambios, frente a los cuales ejecutan ciertas acciones para contrarrestar situaciones que pueden considerarse como amenazantes. Esto se define como métodos de afrontamiento frente a situaciones estresantes. Pueden ser de diversos tipos teniendo en cuenta a la persona y a sus experiencias previas. Asimismo, una posible ruta de escape de dichas situaciones estresantes puede originar lo que se conoce como la orientación suicida, es decir, el hecho de sentirse que no se es útil en la vida o que su vida no tiene importancia para su familia, lo que puede conducir a un intento de suicidio o a consumarlo.

La Institución Educativa Nuestra Señora de Las Mercedes, es una de las instituciones educativas emblemáticas de la ciudad de Ica, que pertenece al estado. Las estudiantes que conforman la población estudiantil de la institución educativa son del sexo femenino. Se seleccionó este tipo de institución teniendo como base que las tasas más elevadas de conductas e ideación suicida se presentan en mujeres en comparación con el sexo masculino (Moscicki, 1995). Además, entre enero del año 2017 y el mes de junio pasado se ha registrado un total de 64 casos de intento de suicidio en el Perú, entre menores cuyas edades varían entre los 8 y 17 años, según el Instituto Nacional de Salud Mental Honorio Delgado - Hideyo Noguchi (INSM). Asimismo, Hirshfled y Russell (1997) encontraron que existe una mayor incidencia con las edades comprendidas entre los 15 y 24 años, debido a eso se seleccionó el grupo correspondiente a los dos últimos años del nivel secundario, ya que se encuentran comprendidos en el primer grupo de incidencia. Además, según investigaciones del INSM la prevalencia de los deseos suicidas en la población adolescente es alta en Lima $29.1 \%$ y en ciudades de la costa $29 \%$ (INSM, 2012).

Durante el trabajo realizado en el Departamento Psicopedagógico en la institución educativa se comprobó que un $45 \%$ de estudiantes que acudieron a consulta presentaban signos de autolesiones, específicamente utilizaban el método del "cutting", el cual consiste en hacerse cortes con una tijera o un objeto afilado en los miembros superiores. Tomando en consideración al suicidio como un proceso dinámico en el cual las conductas se presentan de forma progresiva, la orientación suicida es una condición necesaria para desplegar las acciones suicidas. Maris (1991) considera el método del "cutting" como un predictivo dentro de la orientación suicida, es decir, un comportamiento autoagresivo y que atenta contra la integridad misma de la estudiante. Asimismo, dentro de la consulta se encontró un alto índice de estudiantes que poseían ideación suicida $(35 \%)$, con ideas de desesperanza, autodesprecio entre otros; siendo esto un indicador alarmante.

Cabe resaltar que la decisión de quitarse la vida o la ideación suicida aparece como un comportamiento funcional frente a poner fin a una situación de sufrimiento y tensión que se ha convertido en insoportable para la persona que lo padece (Casullo, Bonaldi y Fernández, 2000). Teniendo en cuenta la realidad descrita, este comportamiento puede darse con más frecuencia de lo que se pueda pensar. Dicha problemática conllevó a plantear una de las variables, que es la orientación suicida.

Se considera la orientación suicida como una respuesta frente a una situación que sobrepasa las barreras de la persona. Asimismo, dentro de la institución educativa se observó una elevada incidencia de peleas entre estudiantes, así como un nivel bajo de tolerancia a la frustración, quienes frente a situaciones estresantes, como la presentación de un trabajo o algún examen de suficiencia académica reaccionaban de manera agresiva. Incluso se observó en algunos casos deserción escolar; cabe resaltar que adicionalmente las estudiantes presentan indicadores de depresión que se evidencia en sus conductas, así como las autolesiones ya mencionadas y una baja percepción de su autoestima, siendo factores de riesgo que pueden desencadenar en una orientación suicida.

Además, las estudiantes pertenecen a un nivel socioeconómico bajo y promedio bajo, lo que ocasiona una serie de vicisitudes como delincuencia, drogas, entre otras, convirtiéndose en factores de riesgo externo, en el contexto familiar. La mayoría proviene de hogares disfuncionales, en donde no se limitan las funciones de los niños y en algunos casos cumplen roles que no les corresponde, propiciando una carga excesiva de estrés. Esto generó la selección de la otra 
variable del presente estudio, que es el afrontamiento al estrés, el cual implica todos los métodos que las estudiantes utilizan para sobreponerse a las situaciones estresantes, y si es que este afrontamiento es adecuado para un desempeño apropiado dentro de la institución educativa, sin caer en conductas autoagresivas u orientaciones suicidas (Vargas \& Saavedra, 2012)

Considerando que el estrés es la interrelación entre la persona y su entorno, De Vera (2004) considera que existe estrés cuando un evento resulta amenazante o desborda los recursos que posee la persona. El afrontamiento al estrés en estudiantes implica tres conjuntos de variables: (a) los estresores o estímulos del entorno educativo experimentados por los estudiantes, como sobrecarga o presión excesiva, (b) las consecuencias del estrés académico sobre la salud o el bienestar psicológico de los estudiantes, sobre su funcionamiento cognitivo o socioafectivo, sobre el rendimiento académico, etc. Y (c) las variables moduladoras o mediadoras del estrés (Muñoz, 2004).

En esa línea, tenemos que las estudiantes presentan además de las características mencionadas, calificaciones que en su mayoría son promedio bajo y promedio, lo que disminuye la cantidad de opciones de ingresar a realizar estudios de nivel superior. Asimismo, el estrés es un tema de interés en la actualidad, tanto en el estudio científico como en el desarrollo de la vida cotidiana, debido a las consecuencias que tiene y el nivel de afectación en la persona en sus diversas realidades (Hobfoll, 1989).

La presente investigación buscó establecer la relación existente entre el afrontamiento al estrés (AE) y la orientación suicida (OS) que presentan las estudiantes de la institución educativa Nuestra Señora de Las Mercedes Los resultados obtenidos pueden servir de base para la elaboración de planes de acción orientados a salvaguardar la integridad de las estudiantes, así como realizar futuras investigaciones de naturaleza explicativa.

\section{MARCO TEÓRICO}

En la presente investigación se utilizará el concepto de estrés psicológico desde un enfoque transaccional del comportamiento, el cual explica que los cambios experimentados por los individuos se interpretan desde la relación entre la persona, el ambiente físico y el ambiente social que lo rodea; es decir no es meramente un producto de un suceso externo o interno del sujeto sino una interpretación de dichos eventos. Asimismo, el afrontamiento es el conjunto de respuestas emocionales, cognitivas y conductuales que median la experiencia de estrés y su finalidad es reducir la respuesta fisiológica y emocional ocasionada; es decir, son las acciones encubiertas o manifiestas que la persona ejecuta para contrarrestar las situaciones que considera amenazantes (Lazarus \& Folkman, 1984).

El afrontamiento es la respuesta adaptativa al estrés, es decir son los esfuerzos cognitivos y comportamentales constantemente cambiantes para manejar las demandas específicas externas o internas apreciadas como excedentes o que desbordan los recursos del individuo (Lazarus \& Folkman, 1984).

Por otro lado, la adaptación hace referencia a la totalidad de la conducta que está dirigida a poder desenvolverse en una nueva situación para lograr su supervivencia. En cambio, en el afrontamiento el objetivo es adquirir cierto control de la situación que se le presenta al individuo (McCrae, 1996).

El afrontamiento forma parte de los recursos psicológicos de los individuos y está relacionado con las emociones que experimenta la persona en un determinado contexto, depende de sus valores, metas y creencias. (Zaldívar, 1996). Las estrategias de afrontamiento son eficaces si contribuyen al bienestar fisiológico, psicológico y social de la persona. Las cuales se pueden reflejar en la capacidad para controlar y reducir el malestar emocional y sus efectos a largo plazo ocasionados en la persona (Snyder, 1999).

Dimensiones del afrontamiento al estrés según Carver (1984):

a) Afrontamiento activo: hace referencia a tomar acciones concretas dirigidas a disminuir la situación estresante.

b) Planificación: hace referencia a la elaboración de actividades planificadas dirigidas a afrontar la situación estresante. 
c) Supresión de otras actividades: hace referencia a la eliminación de actividades para enfocarse en la situación estresante.

d) Postergación del afrontamiento: hace referencia al aplazamiento de afrontar la situación estresante.

e) Búsqueda de apoyo social: hace referencia a buscar soporte emocional en la interacción con las personas que le rodean.

f) Búsqueda de soporte emocional: hace referencia a la búsqueda de aceptación y apoyo emocional por parte de personas cercanas o familiares

g) Reinterpretación positiva y desarrollo personal: hace referencia a dar una nueva interpretación a los eventos estresantes logrando que su interpretación no genere estrés en la persona.

h) Aceptación: hace referencia a afrontar y reconocer como tal la situación generadora de estrés.

i) Acudir a la religión: hace referencia a apelar a la parte espiritual de la persona, encomendándose a un ser superior quien le brinda seguridad.

j) Análisis de las emociones: hace referencia a analizar de manera racional las emociones, utilizando la inteligencia emocional.

k) Negación: hace referencia a negar la realidad de la situación estresante.

1) Conductas inadecuadas: hace referencia a realizar conductas disruptivas como medio de desfogar el estrés causado por algún evento.

m) Distracciones: hace referencia prestar atención a otras actividades para no enfocarse en la situación estresante en sí.

El suicidio en la juventud es un fenómeno preocupante para la sociedad en general y para los profesionales que trabajan dentro de las instituciones educativas, debido a que se considera como un problema de salud pública (OMS, 2009). Asimismo, el suicidio tiene un impacto profundo en el área emocional en al menos seis personas que pertenezcan al círculo cercano de quien lo comete, por lo tanto, de ocurrir un suicidio en una institución educativa el impacto es mayor debido a la cantidad de personas que pertenecen a su entorno (Organización Mundial de la Salud - OMS, 2000). En esta investigación se abordará la ideación suicida desde la perspectiva de un proceso dinámico que se desencadena a nivel multifactorial y desencadena en el suicidio.

En Perú, se han encontrado que los estudiantes cuyas edades fluctúan entre los 15 y 24 años son los de mayor incidencia con ideación suicida, ha sido relacionada con la baja cohesión familiar y problemas dentro de la institución educativa (Caycedo et al., 2010)

Asimismo, según investigaciones se han encontrado que las características socioculturales relacionadas con la orientación suicida en estudiantes incluyen niveles educativos medios o bajos, residencia en zonas rurales y de condición económica deficiente (Manrique et al., 2002).

Por otro lado, algunas de las características psicológicas fueron antecedentes psicopatológicos previos, trastorno de conducta disocial y depresión. Cabe resaltar que la depresión no se puede considerar como el único factor asociado debido al carácter multifactorial y al proceso dinámico del suicidio (Barrera et al., 2007).

Pérez (1999) considera que el suicido es un proceso compuesto por diversas acciones, se inicia con la ideación suicida- Por ello, es importante abordar esta variable. En la misma línea, estudios definen al suicido como un proceso que inicia con la ideación suicida y luego pasa a la tentativa o intento suicida para finalmente acabar en la muerte (González et al., 2003)

Dimensiones de la orientación suicida según Casullo \& Fernández (2007):

a) Desesperanza: considerada como una actitud negativa y pesimista acerca de la vida, carente de esperanza y sin motivación alguna.

b) Baja autoestima: considerada como una valoración negativa de sí mismo.

c) Incapacidad para afrontar emociones: falta de capacidad para poder tolerar el rechazo, poca tolerancia a la frustración, incapacidad de afronte. 
d) Soledad y abatimiento: sentimientos de estar solo, incomprendido acompañado de tristeza o melancolía; falta de ánimo o energía.

e) Ideación suicida: pensamientos recurrentes en torno a quitarse la vida.

\section{METODOLOGÍA}

La investigación es de tipo cuantitativo, no experimental y correlacional. Es cuantitativa, ya que usa la recolección de datos para probar hipótesis, con base en la medición numérica y el análisis estadístico, para establecer patrones de comportamiento y probar teorías (Hernández, Fernández y Baptista, 2006). Es no experimental, debido a que el investigador observa los fenómenos tal y como ocurren naturalmente, sin intervenir en su desarrollo (Behar, 2008). Es correlacional, ya que se persigue fundamentalmente determinar el grado en el cual las variaciones en uno o varios factores son concomitantes con la variación en otro u otros factores. La existencia y fuerza de esta covariaciónnormalmente se determinaestadísticamente por medio de coeficientes de correlación. Es conveniente tener en cuenta que esta covariación no significa que entre los valores existan relaciones de causalidad (Tamayo, 2003). Es importante señalar que los padres dieron el consentimiento informado para la presente investigación.

La población que es motivo de estudio estuvo conformada por 180 estudiantes de la Institución Educativa Nuestra Señora de Las Mercedes de la ciudad de Ica. En este caso por ser una cantidad de población reducida se utilizó la muestra censal. Las edades de las estudiantes de sexo femenino fluctúan entre los 15 y 17 años, que se encuentran cursando el $4^{\circ}$ y $5^{\circ}$ año de nivel secundaria.

\section{INSTRUMENTOS}

La variable afrontamiento al estrés utilizó el Cuestionario de Modos de Afrontamiento al Estrés (COPE), elaborada en 1989 por Carver, Sheir y Weintraub, la versión del instrumento que se utilizó fue la adaptación realizada por Guevara, Hernández y Flores en 2001, en un estudio conformado por 88 pacientes que se encontraban recibiendo tratamiento en Comunidades Terapéuticas de Lima, donde se estableció la confiabilidad mediante el estadístico de
Alfa de Cronbach, con valores de 0.84 en la escala de acudir a la religión, 0,7 en afrontamiento activo y 0.78 en búsqueda de apoyo social, con lo cual se demuestra la confiabilidad del cuestionario. El cuestionario está conformado por 52 preguntas y se divide en 3 áreas: estilos de afrontamiento enfocados en el problema, en la emoción y otros estilos adicionales. Asimismo, posee 13 sub-dimensiones con escalas tipo Likert con valores del 1 a 4. Su administración puede ser de manera individual o grupal. En este estudio, se obtuvo una confiabilidad de ,710 (Alfa de Cronbach) lo cual indica una consistencia interna aceptable para esta escala.

La variable orientaciones suicidas utilizó el Inventario de Orientaciones Suicidas (IOS), el cual fue elaborado en 1994 por King y Kowalchuk, la versión que se utilizó en la presente investigación fue la adaptada para toda Sudamérica por Casullo y Fernández en 2007. Para la validez del instrumento se utilizó el alfa de Cronbach obteniendo un puntaje de 0.779 corroborando de esa manera la validez. En el test re-test se obtuvo una correlación de 0.86 . Asimismo, el instrumento está conformado por 30 ítems que evalúan cinco variables psicológicas implicadas en la orientación suicida, las cuales son: desesperanza, baja autoestima, incapacidad para afrontar emociones, soledad y abatimiento e ideación suicida. En este estudio, se obtuvo una confiabilidad de ,901 (Alfa de Cronbach) la cual indica una buena consistencia interna para esta escala. Cabe resaltar que el presente instrumento ha sido utilizado en población peruana con estudiantes de una institución educativa en Arequipa por Anculle, 2016.

\section{PROCEDIMIENTO}

La aplicación de los instrumentos psicológicos fue de manera colectiva y los resultados obtenidos fueron ingresados a una base de datos en el programa estadístico SPSS versión 24 para su correspondiente tratamiento estadístico, en el cual se utilizó el coeficiente de correlación de Pearson. Asimismo, los resultados obtenidos se presentan en tablas para una mejor apreciación y comprensión de los lectores.

\section{RESULTADOS}

La distribución de la muestra fue de la siguiente manera como se puede apreciar en la siguiente tabla: 
Tabla 1

Distribución de la muestra

\begin{tabular}{lrrrr}
\hline & Frecuencia & Porcentaje & $\begin{array}{c}\text { Porcentaje } \\
\text { válido }\end{array}$ & $\begin{array}{r}\text { Porcentaje } \\
\text { acumulado }\end{array}$ \\
\hline $4^{\circ}$ año de secundaria & 100 & $55.6 \%$ & $55.6 \%$ & $55.6 \%$ \\
$5^{\circ}$ año de secundaria & 80 & $44,4 \%$ & $44.4 \%$ & $100.0 \%$ \\
Total & 180 & $100.0 \%$ & & \\
\hline
\end{tabular}

Se puede observar en la tabla 1 que la muestra evaluada estuvo conformada por 180 estudiantes, de las cuales 100 que corresponde al $55.6 \%$ pertenecían al $4^{\circ}$ año de secundaria y 80 que corresponde al $44.4 \%$ pertenecían al $5^{\circ}$ año de secundaria.

A continuación, se muestran los resultados obtenidos de las correlaciones entre el afrontamiento al estrés y la orientación suicida en las estudiantes de una institución educativa del departamento de Ica.

Tabla 2

Correlación entre afrontamiento al estrés y orientación suicida

\begin{tabular}{llrr}
\hline & & $\begin{array}{c}\text { Orientación } \\
\text { suicida }\end{array}$ & $\begin{array}{c}\text { Afrontamiento } \\
\text { al estrés }\end{array}$ \\
\hline Afrontamiento al estrés & C. Pearson &,$- 292^{* *}$ & 1 \\
& Sig. (bilateral) &, 000 & \\
Orientación suicida & C. Pearson & 1 &,$- 292^{* *}$ \\
& Sig. (bilateral) & &, 000 \\
\hline
\end{tabular}

**La correlación es significativa en el nivel 0.01 (bilateral)

En la tabla 2, se aprecia la correlación obtenida al aplicar los instrumentos psicológicos sobre las variables afrontamiento al estrés y orientación suicida. Se encontró que existe una correlación muy significativa y negativa, con un coeficiente de correlación de Pearson de -,292.
Tabla 3 .

Correlación afrontamiento al estrés y desesperanza

\begin{tabular}{|c|c|c|}
\hline $\begin{array}{l}\text { Dimensiones de modo } \\
\text { de afrontamiento al estrés }\end{array}$ & & $\begin{array}{l}\text { orientación suicida } \\
\text { esperanza }\end{array}$ \\
\hline Afrontamiento activo & $\begin{array}{l}\text { C. Pearson } \\
\text { Sig. }\end{array}$ & $\begin{array}{l}-, 221 * * \\
, 003\end{array}$ \\
\hline Planificación & $\begin{array}{l}\text { C. Pearson } \\
\text { Sig. }\end{array}$ & $\begin{array}{l}-, 081 \\
, 279\end{array}$ \\
\hline $\begin{array}{l}\text { Supresión de otras } \\
\text { actividades }\end{array}$ & $\begin{array}{l}\text { C. Pearson } \\
\text { Sig. }\end{array}$ & $\begin{array}{l}-, 067 \\
, 368\end{array}$ \\
\hline $\begin{array}{l}\text { Postergación del } \\
\text { afrontamiento }\end{array}$ & $\begin{array}{l}\text { C. Pearson } \\
\text { Sig. }\end{array}$ & $\begin{array}{l}-, 107 \\
, 154\end{array}$ \\
\hline Búsqueda de apoyo social & $\begin{array}{l}\text { C. Pearson } \\
\text { Sig. }\end{array}$ & $\begin{array}{l}-, 137 \\
, 066\end{array}$ \\
\hline $\begin{array}{l}\text { Búsqueda de soporte } \\
\text { emocional }\end{array}$ & $\begin{array}{l}\text { C. Pearson } \\
\text { Sig. }\end{array}$ & $\begin{array}{l}-, 118 \\
, 116\end{array}$ \\
\hline $\begin{array}{l}\text { Reinterpretación positiva } \\
\text { y desarrollo personal }\end{array}$ & $\begin{array}{l}\text { C. Pearson } \\
\text { Sig. }\end{array}$ & $\begin{array}{l}-, 120 \\
, 109\end{array}$ \\
\hline Aceptación & $\begin{array}{l}\text { C. Pearson } \\
\text { Sig. }\end{array}$ & $\begin{array}{l}-, 052 \\
, 490\end{array}$ \\
\hline Acudir a la religión & $\begin{array}{l}\text { C. Pearson } \\
\text { Sig. }\end{array}$ & $\begin{array}{l}-, 117 \\
, 118\end{array}$ \\
\hline Análisis de las emociones & $\begin{array}{l}\text { C. Pearson } \\
\text { Sig. }\end{array}$ & $\begin{array}{l}-, 127 \\
, 088\end{array}$ \\
\hline Negación & $\begin{array}{l}\text { C. Pearson } \\
\text { Sig. }\end{array}$ & $\begin{array}{l}-, 069 \\
, 360\end{array}$ \\
\hline Conductas inadecuadas & $\begin{array}{l}\text { C. Pearson } \\
\text { Sig. }\end{array}$ & $\begin{array}{l}-, 183^{*} \\
, 014\end{array}$ \\
\hline Distracción & $\begin{array}{l}\text { C. Pearson } \\
\text { Sig. }\end{array}$ & $\begin{array}{l}-, 017 \\
, 824\end{array}$ \\
\hline
\end{tabular}

**La correlación es significativa en el nivel 0.01 (bilateral)

En la tabla 3, se aprecia las correlaciones obtenidas entre las variables afrontamiento al estrés y la dimensión desesperanza de la orientación suicida. Se obtuvo una correlación muy significativa y negativa entre el afrontamiento activo y la desesperanza.

En las dimensiones de planificación, supresión de otras actividades, postergación del afrontamiento, búsqueda de apoyo social, búsqueda de soporte emocional, reinterpretación positiva y desarrollo personal, aceptación, acudir a la religión, análisis de las emociones, negación y distracción se encontró una relación negativa con la dimensión desesperanza de la orientación suicida. 
Finalmente, en la dimensión de conductas inadecuadas se muestra una relación significativa, negativa con la dimensión desesperanza. Por lo tanto, se observa que existe una relación negativa entre las dimensiones del afrontamiento al estrés y la dimensión desesperanza de la orientación suicida.

Tabla 4.

Correlación afrontamiento al estrés y baja autoestima

\begin{tabular}{|c|c|c|}
\hline \multicolumn{2}{|l|}{$\begin{array}{l}\text { Dimensiones de modo de } \\
\text { afrontamiento al estrés }\end{array}$} & \multirow{2}{*}{$\begin{array}{c}\text { Dimensión de orientación suicida } \\
\text { Baja autoestima } \\
-, 241^{* *}\end{array}$} \\
\hline Afrontamiento activo & $\begin{array}{l}\text { C. Pearson } \\
\text { Sig. }\end{array}$ & \\
\hline Planificación & $\begin{array}{l}\text { C. Pearson } \\
\text { Sig. }\end{array}$ & $\begin{array}{r}-, 089 \\
, 236\end{array}$ \\
\hline $\begin{array}{l}\text { Supresión de otras } \\
\text { actividades }\end{array}$ & $\begin{array}{l}\text { C. Pearson } \\
\text { Sig. }\end{array}$ & $\begin{array}{r}-, 044 \\
, 559\end{array}$ \\
\hline $\begin{array}{l}\text { Postergación del } \\
\text { afrontamiento }\end{array}$ & $\begin{array}{l}\text { C. Pearson } \\
\text { Sig. }\end{array}$ & $\begin{array}{r}-, 112 \\
, 135\end{array}$ \\
\hline Búsqueda de apoyo social & $\begin{array}{l}\text { C. Pearson } \\
\text { Sig. }\end{array}$ & $\begin{array}{r}-, 171 * \\
, 022\end{array}$ \\
\hline $\begin{array}{l}\text { Búsqueda de soporte } \\
\text { emocional }\end{array}$ & $\begin{array}{l}\text { C. Pearson } \\
\text { Sig. }\end{array}$ & $\begin{array}{r}-, 180 * \\
, 016\end{array}$ \\
\hline $\begin{array}{l}\text { Reinterpretación positiva } \\
\text { y desarrollo personal }\end{array}$ & $\begin{array}{l}\text { C. Pearson } \\
\text { Sig. }\end{array}$ & $\begin{array}{r}-, 150 * \\
, 045\end{array}$ \\
\hline Aceptación & $\begin{array}{l}\text { C. Pearson } \\
\text { Sig. }\end{array}$ & $\begin{array}{r}-, 020 \\
, 785\end{array}$ \\
\hline Acudir a la religión & $\begin{array}{l}\text { C. Pearson } \\
\text { Sig. }\end{array}$ & $\begin{array}{r}-, 073 \\
, 333\end{array}$ \\
\hline Análisis de las emociones & $\begin{array}{l}\text { C. Pearson } \\
\text { Sig. }\end{array}$ & $\begin{array}{r}-, 177 * \\
, 017\end{array}$ \\
\hline Negación & $\begin{array}{l}\text { C. Pearson } \\
\text { Sig. }\end{array}$ & $\begin{array}{r}-, 090 \\
, 229\end{array}$ \\
\hline Conductas inadecuadas & $\begin{array}{l}\text { C. Pearson } \\
\text { Sig. }\end{array}$ & $\begin{array}{r}-, 196 * * \\
, 008\end{array}$ \\
\hline Distracción & $\begin{array}{l}\text { C. Pearson } \\
\text { Sig. }\end{array}$ & $\begin{array}{r}-, 068 \\
, 363\end{array}$ \\
\hline
\end{tabular}

**La correlación es significativa en el nivel 0.01 (bilateral)

En la tabla 4, se aprecian las correlaciones obtenidas entre las variables afrontamiento al estrés y la dimensión baja autoestima de la orientación suicida. Se obtuvo una correlación muy significativa y negativa entre el afrontamiento activo y la baja autoestima. Así como en la dimensión conductas inadecuadas, que también se encontró una relación muy significativa y negativa.

En las dimensiones de planificación, supresión de otras actividades, postergación del afrontamiento, aceptación, acudir a la religión, negación y distracción se encontró una relación negativa con la dimensión baja autoestima de la orientación suicida.

Finalmente, en la dimensión de búsqueda de apoyo social, búsqueda de soporte emocional, reinterpretación positiva, y desarrollo personal y análisis de las emociones se muestra una relación significativa, negativa con la dimensión baja autoestima. Por lo tanto, se observa que existe una relación negativa entre las dimensiones del afrontamiento al estrés y la dimensión baja autoestima de la orientación suicida.

Tabla 5

Correlación afrontamiento al estrés e incapacidad para afrontar emociones

\begin{tabular}{llr}
\hline $\begin{array}{l}\text { Dimensiones de modo de } \\
\text { afrontamiento al estrés }\end{array}$ & \multicolumn{1}{c}{$\begin{array}{c}\text { Dimensión de orientación suicida } \\
\text { Incapacidad para afrontar emociones }\end{array}$} \\
\hline Afrontamiento activo & C. Pearson &,- 144 \\
& Sig. &, 054 \\
Planificación & C. Pearson &,$- 166^{*}$ \\
Supresión de otras & Sig. &, 026 \\
actividades & C. Pearson &,$- 148^{*}$ \\
Postergación del & Sig. &, 048 \\
afrontamiento & C. Pearson &,- 108 \\
& Sig. &, 151 \\
Búsqueda de apoyo social & C. Pearson &,$- 227^{* *}$ \\
& Sig. &, 002 \\
Búsqueda de soporte & C. Pearson &,$- 189^{*}$ \\
emocional & Sig. &, 011 \\
Reinterpretación positiva & C. Pearson &,- 084 \\
y desarrollo personal & Sig. &, 260 \\
Aceptación & C. Pearson &,- 072 \\
& Sig. &, 340 \\
Acudir a la religión & C. Pearson &,$- 174^{*}$ \\
& Sig. &, 020 \\
Análisis de las emociones & C. Pearson &,- 111 \\
Negación & Sig. &, 136 \\
Conductas inadecuadas & C. Pearson &,- 032 \\
Distracción & Cig. Pearson &, 668 \\
& Sig. &,$- 158^{*}$ \\
& C. Pearson &, 004 \\
\hline & Sig. & \\
\hline
\end{tabular}

**La correlación es significativa en el nivel 0.01 (bilateral)

En la tabla 5, se aprecian las correlaciones obtenidas entre las variables afrontamiento al estrés y la dimensión incapacidad para afrontar emociones de la orientación suicida. Se obtuvo una correlación muy significativa y negativa entre la búsqueda de apoyo social y la incapacidad para afrontar emociones. 
En las dimensiones de postergación del afrontamiento, reinterpretación positiva y desarrollo personal, aceptación, análisis de las emociones, negación y distracción se encontró una relación negativa con la dimensión incapacidad para afrontar emociones de la orientación suicida.

Finalmente, en la dimensión planificación, supresión de otras actividades, búsqueda de soporte emocional, acudir a la religión y conductas inadecuadas se muestra una relación significativa y negativa con la dimensión baja autoestima. Por lo tanto, se observa que existe una relación negativa entre las dimensiones del afrontamiento al estrés y la dimensión incapacidad para afrontar emociones de la orientación suicida.

Tabla 6.

Correlación afrontamiento al estrés y soledad y abatimiento

\begin{tabular}{llc}
\hline $\begin{array}{l}\text { Dimensiones de modo de } \\
\text { afrontamiento al estrés }\end{array}$ & $\begin{array}{c}\text { Dimensión de orientación suicida } \\
\text { Soledad y abatimiento }\end{array}$ \\
\hline Afrontamiento activo & C. Pearson &,$- 231^{* *}$ \\
& Sig. &, 002 \\
Planificación & C. Pearson &,- 070 \\
Supresión de otras & Sig. &, 351 \\
actividades & C. Pearson &,- 073 \\
Postergación del & Sig. &, 331 \\
afrontamiento & C. Pearson &,- 116 \\
Búsqueda de apoyo social & Sig. &, 122 \\
Búsqueda de soporte & Sig. &,- 125 \\
emocional & C. Pearson &, 095 \\
Reinterpretación positiva & Sig. &,- 110 \\
y desarrollo personal & C. Pearson &, 140 \\
& Sig. &,- 114 \\
Aceptación & C. Pearson &, 129 \\
& Sig. &,- 039 \\
Acudir a la religión & C. Pearson &, 602 \\
& Sig. &,- 102 \\
Análisis de las emociones & C. Pearson &, 174 \\
Negación & Sig. &,- 123 \\
Conductas inadecuadas & C. Pearson &,- 044 \\
Distracción & Sig. &, 560 \\
& C. Pearson &,$- 189 *$ \\
& Sig. &,- 006 \\
\hline
\end{tabular}

**La correlación es significativa en el nivel 0.01 (bilateral)

En la tabla 6, se aprecian las correlaciones obtenidas entre las variables afrontamiento al estrés y la dimensión soledad y abatimiento de la orientación suicida. Existiendo una correlación muy significativa y negativa entre el afrontamiento activo y la soledad y abatimiento.

En las dimensiones de planificación, supresión de otras actividades, postergación del afrontamiento, búsqueda de apoyo social, búsqueda de soporte emocional, reinterpretación positiva y desarrollo personal, aceptación, acudir a la religión, análisis de las emociones, negación y distracción se encontró una relación negativa con la dimensión baja autoestima de la orientación suicida.

Finalmente, en la dimensión de conductas inadecuadas se muestra una relación significativa y negativa con la dimensión soledad y abatimiento. Por lo tanto, se observa que existe una relación negativa entre las dimensiones del afrontamiento al estrés y la dimensión soledad y abatimiento de la orientación suicida.

Tabla 7.

Correlación afrontamiento a estrés e ideación suicida

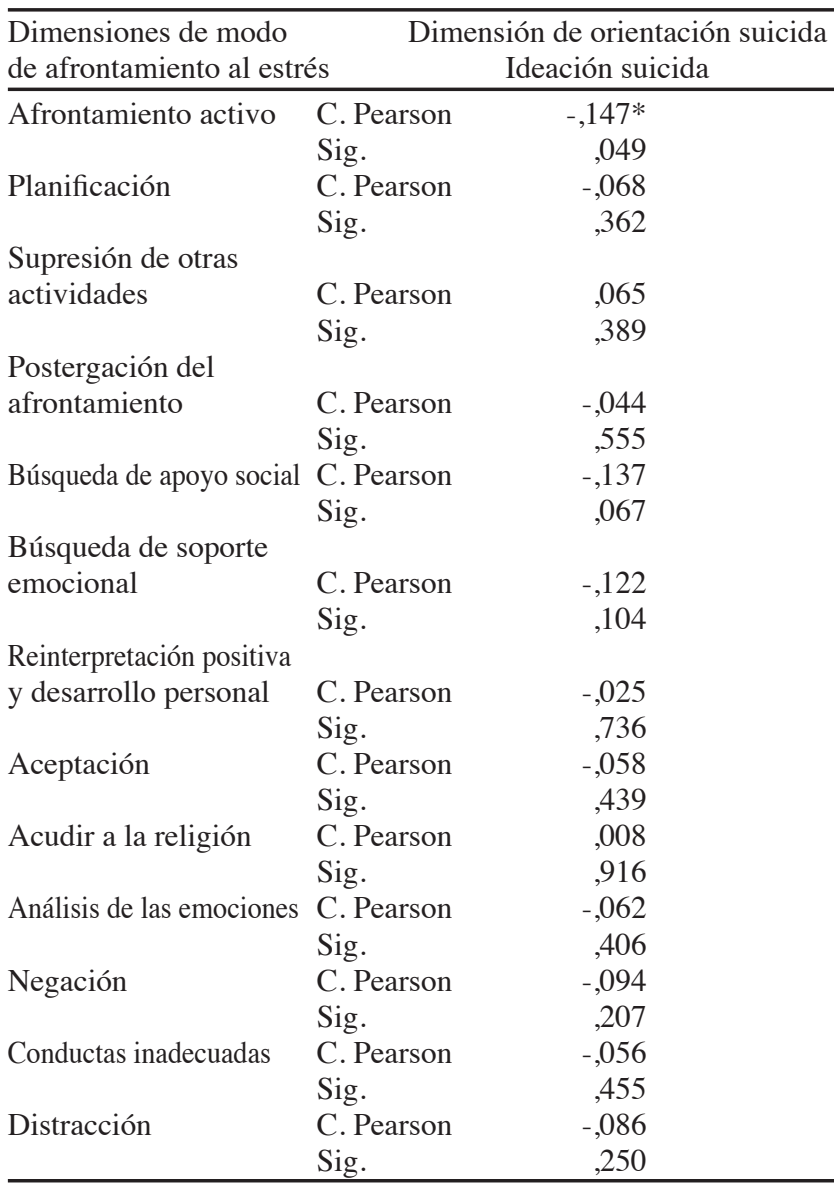

**La correlación es significativa en el nivel 0.01 (bilateral) 
En la tabla 7, se aprecian las correlaciones obtenidas entre las variables afrontamiento al estrés y la dimensión ideación suicida de la orientación suicida. Existiendo una correlación significativa y negativa entre el afrontamiento activo y la ideación suicida.

En las dimensiones de planificación, postergación del afrontamiento, búsqueda de apoyo social, búsqueda de soporte emocional, reinterpretación positiva y desarrollo personal, aceptación, análisis de las emociones y negación se encontró una relación negativa con la dimensión baja autoestima de la orientación suicida.

Finalmente, en la dimensión de supresión de otras actividades y acudir a la religión muestran una relación positiva con la dimensión ideación suicida. Por lo tanto, se observa que existe una relación inversa entre la mayoría de las dimensiones del afrontamiento al estrés y la dimensión ideación suicida de la orientación suicida.

\section{DISCUSIÓN}

En el análisis, se aprecia, que ambos instrumentos fueron aplicados a 180 estudiantes del nivel secundaria, de los cuales 100 estudiantes pertenecen $4^{\circ}$ grado de secundaria y 80 estudiantes al $5^{\circ}$ grado de secundaria. Se puede apreciar que la mayoría de las encuestadas están cursando el $4^{\circ}$ grado de secundaria $(55,55 \%)$.

Cabe resaltar, que la incidencia sobre la orientación suicida es mayor en mujeres que en hombres, comprobando lo propuesto por Anculle (2016); por lo cual se seleccionó una institución educativa de mujeres

Respecto a la edad de los las estudiantes del nivel secundaria se encontró que 82 estudiantes tienen 15 años de edad; 70 poseen 16 años y 28 estudiantes tienen 17 años de edad, por lo que se puede apreciar que mayoría de las encuestadas tienen 15 años de edad. Además, las evaluadas provienen de familias donde su funcionamiento familiar carece de límites y puede encontrarse un riesgo suicida a nivel moderado en dicha población, según lo propuesto por Anculle (2016) quien considera que este tipo de funcionamiento familiar con límites difusos, denominado de tipo aglutinado según el modelo de Olson (1989) es uno de los influyentes de forma negativa sobre la orientación suicida.

Además, se comprueba con la correlación significativa negativa lo propuesto por Paniagua, Gonzales y Rueda (2014) quienes consideraron que la acumulación de tensiones en la familia y el escaso apoyo social podrían desencadenar un mayor riesgo de orientación suicida, lo que ocurre en la presente investigación debido al entorno familiar de las estudiantes. Sin embargo, en línea con lo propuesto por Amoretti (2017) quien afirma que los adolescentes tienden a tener un nivel promedio de afrontamiento al estrés mediante otros modos. En el caso de nuestra investigación, las estudiantes presentan un afrontamiento activo, pues el 53\% de las evaluadas reflejan que enfrenta al estrés de manera frecuente; esto sirve como un factor protector frente al desencadenamiento de una orientación suicida en ellas.

Cabe resaltar, que en consonancia con Serrano y Flores (2005) a mayor cantidad de estrés en mujeres, existe mayor probabilidad de aumento de ideación suicida, sin embargo, depende de los métodos de afrontamiento que utilicen las personas, lo cual puede disminuir dicho riesgo. En la misma línea, Prince (2016) encontró que cuando existe una mayor dificultad para afrontar el estrés los adolescentes tienden a actuar con mayor agresividad, la que puede ser dirigida a sí mismo. Esto conlleva a la hipótesis planteada, que a mayor afrontamiento al estrés disminuye la orientación suicida en estudiantes, lo que se ha demostrado en la presente investigación.

En una de las investigaciones previas realizadas por Verdugo et al. (2012), encontraron que la aceptación de la responsabilidad era uno de los métodos de afrontamientos más usados, en este caso se encontró que el método afrontamiento activo fue el más utilizado.

\section{CONCLUSIONES}

Luego del análisis de resultados presentamos las siguientes conclusiones:

1. Existe una relación muy significativa y negativa entre la variable afrontamiento al estrés y la 
variable orientación suicida (r: -0.292) en estudiantes de una institución educativa del Departamento de Ica- Perú.

2. Existe una relación negativa en todas las dimensiones del afrontamiento al estrés y la dimensión desesperanza de la variable orientación suicida en estudiantes de una institución educativa del Departamento de IcaPerú. Resalta como muy significativa la relación negativa de la dimensión afrontamiento activo (r: -0.221) y relación significativa en conductas inadecuadas (r: -0.183).

3. Existe una relación negativa entre las dimensiones del afrontamiento al estrés y la dimensión baja autoestima de la variable orientación suicida. En la cual resalta el afrontamiento activo ( $\mathrm{r}$ : -0.241) y las conductas inadecuadas (r: -0.196) con una relación muy significativa; así como la búsqueda de apoyo social (r: -0.171), reinterpretación positiva y desarrollo personal (r: -0.150) y análisis de las emociones (r: -0.177) con una relación significativa y negativa en estudiantes de una institución educativa del Departamento de IcaPerú.

4. Existe una relación negativa entre las dimensiones del afrontamiento al estrés y la dimensión incapacidad para afrontar emociones de la variable orientación suicida en estudiantes de una institución educativa del Departamento de Ica- Perú. Resaltando con una relación muy significativa la búsqueda de apoyo social (r: -0.277). Además, presenta una relación y negativa con las dimensiones de planificación (r: -0.166), búsqueda de soporte emocional (r: -0.189), acudir a la religión ( $\mathrm{r}$ : -0.174 ) y conductas inadecuadas (r: -0.158).

5. Existe una relación negativa entre las dimensiones afrontamiento al estrés y la dimensión soledad y abatimiento de la variable orientación suicida en estudiantes de una institución educativa del Departamento de Ica- Perú. Resaltando una relación muy significativa con la dimensión afrontamiento activo (r: -0.231) y significativa con la dimensión conductas inadecuadas (r: -0.189).
6. Existe una relación negativa entre las dimensiones afrontamiento al estrés y la dimensión ideación suicida de la variable orientación suicida en estudiantes de una institución educativa del Departamento de IcaPerú. Resaltando una relación significativa con la dimensión afrontamiento activo (r: -0.147).

\section{REFERENCIAS}

Amoretti, T. (2017). Ansiedad frente a exámenes y afrontamiento de estrés en adolescentes de un centro educativo de Villa el Salvador (Tesis de licenciatura). Universidad Autónoma del Perú, Lima.

Anculle, M. (2016). Influencia del funcionamiento familiar en la orientación suicida de los estudiantes de la Institución Educativa Manuel Muñoz Najar y la Institución Educativa Micaela Bastidas, Arequipa (Tesis de licenciatura). Universidad Alas Peruanas, Arequipa.

Barrera, L., Ordóñez, R., Palacios, E., \& Peña, A. (2007). Análisis Bibliométrico de la Producción Científica sobre suicidio en niños en el periodo 1985-2005. Revista Avances en Psicología Latinoamericana, 25(2), 40-62.

Behar, D. (2008). Metodología de la Investigación. Colombia: Shalom.

Caycedo, A., Arenas, M., Benítez, M., Cavanzo, P., Leal, G., y Guzmán, Y. (2010). Características psicosociales y familiares relacionadas con intento de suicidio en una población adolescente en Bogotá - 2009. Persona Bioética. Recuperado de http://redalyc.uaemex.mx/src/inicio/ArtPdfRed. jsp?iCve $=83216977008$.

Carver, R. (1984). Manual del cuestionario de afrontamiento al estrés. Washington: QM Editorial

Carver, C., Scheier, M., \& Weintraub, J. (1989). Evaluar estrategias de afrontamiento: un enfoque teórico. Revista de Personalidad y Psicología Social, 56(2), 267- 283.

Casullo, M., Bonaldi, P., \& Fernández, M. (2000). Comportamientos suicidas en la adolescencia. Morir antes de la Muerte. Buenos Aires: Córdova 
Casullo, M., \& Fernández, L. (2007). Estudiantes adolescentes argentinos en riesgo suicida: una investigación comparativa. Revista Ciencias Psicológicas, 1(1), 37- 45.

De Vera, M. (2004). La teoría del afrontamiento del estrés de Lazarus y los moldes cognitivos (Tesis de doctorado). Universidad de La Laguna, España.

González, C., Mariño, M., Rojas, E., Mondragón, L., \& Medina, M. (2003). Intento de suicidio en estudiantes de la Ciudad de Pachuca, Hgo. y su relación con el malestar depresivo y el uso de sustancias. Revista Mexicana de Psicología, 15(2), 165-175.

Guevara, G., Hernández, H., \& Flores, T. (2001). Estilos de afrontamiento al estrés en pacientes drogodependientes. Revista de Investigación en Psicología, 4(1). 53- 65

Hernández, R., Fernández, C., \& Baptista, M. (2006). Metodología de la investigación. Cuarta edición. México: McGrawHill.

Hirshfled, R. \& Russel, J. (1997). Assesment and treatment of suicidal patients. The New England Journal of Medicine, 337(13), 910- 915.

Hobfoll, S. (1989). Conservation of resources: a new attempt at conceptualizing stress. American Psyuchologist, 44(3), 513- 524

Instituto Nacional de Salud Mental (2012). Estudio epidemiológico de salud mental de niños y adolescentes en Lima Metropolitana y Callao 2007. Informe General. Anales de Salud Mental, 28(1), 1- 220.

King, J. \& Kowalchuk, B. (1994). ISO- 30. Adolescent Inventory of Suicide Orientation-30. Minneapolis: National Computer Systems

Lazarus, R. \& Folkman, S. (1984). Stress, appraisal and coping. Nueva York: Springer

Manrique, R., Ochoa, F., Fernández, L., Escamilla, R., \& Vélez, E. (2002). Comportamiento del suicidio en Antioquia 1998-2000. Revista CES Medicina, 16(1), 7-17.

Maris, R. (1991). Suicide and life threatening behavior. Introduction. New York, Suicide and life threatening behavior, 21(1), 1- 17
McCrae,R.(1996). Social consequences of experiential openness. Psychological Bulletin, 120(1), 323337.

Moscicki, E. (1995). Epidemiology of suicidal behavior. New York, Suicide and Life Threat Behavior, 25(1), 22-35.

Muñoz, F. (2004). El estrés académico. Problemas $y$ soluciones desde una perspectiva psicosocial. España: Servicio de Publicaciones de la Universidad de Huelva

Olson, H. (1989). Circumplex model: systemic assessment and treatment of families. New York: Taylor and Francis.

Organización Mundial de la Salud (2000). Prevención del suicidio. Un instrumento para trabajadores de atención primaria en salud. Recuperado de International Journal of Adolescent Medicine and Health, 19(1), 11- 18.

Organización Mundial de la Salud (2009). Día mundial para le prevención del suicidio. Recuperado de http://www.who.int/mediacentre/news/ releases/2004/pr61/es/index.html

Paniagua, R., Gonzáles, C. \& Rueda, S. (2014). Orientación al suicidio en adolescentes en una zona de Medellín, Colombia. Revista Facultad Nacional de Salud Pública, 32(3), 314-321.

Pérez, S. (1999). El suicidio, comportamiento y prevención. Revista cubana de medicina general, 15(2), 196-217.

Prince, M. (2016). Modos de afrontamiento al estrés $y$ agresividad en estudiantes de secundaria de las instituciones educativas de Nuevo Chimbote (Tesis de licenciatura). Universidad César Vallejo, Nuevo Chimbote.

Serrano, M. \& Flores, M. (2005). Estrés, respuestas de afrontamiento e ideación suicida en adolescentes. Revista Psicología y Salud, 15(2), 221-230.

Snyder, C. R. (1999). The psychology of what works. New York: Oxford University Press

Tamayo, M. (2003). El proceso de la investigación científica. México: Editorial Limusa. 
Vargas, H. \& Saavedra, J. (2012). Factores asociados con la conducta suicida en adolescentes. Revista Neuropsiquiatria, 75(1), 19- 28.

Verdugo, J., Ponce de León, B., Guardado, R., Meda, R., Uribe, J. \& Guzmán, J. (2012). Estilos de afrontamiento al estrés y bienestar subjetivo en adolescentes y jóvenes. Revista Latinoamericana de Ciencias Sociales, Niñez y Juventud, 11(1), 7991.

Zaldívar, D. (1996). Conocimiento y dominio del estrés. La Habana: Editorial Científico- Técnica.

Fecha de Recepción: 14 de febrero 2020

Fecha de Aceptación: 5 de marzo del 2020 